\title{
OPTIMAL SEQUENTIAL CHANGE DETECTION FOR FRACTIONAL DIFFUSION-TYPE PROCESSES
}

\author{
ALEXANDRA CHRONOPOULOU, ${ }^{*}$ University of California, Santa Barbara \\ GEORGIOS FELLOURIS, ${ }^{* *}$ University of Southern California
}

\begin{abstract}
The problem of detecting an abrupt change in the distribution of an arbitrary, sequentially observed, continuous-path stochastic process is considered and the optimality of the CUSUM test is established with respect to a modified version of Lorden's criterion. We apply this result to the case that a random drift emerges in a fractional Brownian motion and we show that the CUSUM test optimizes Lorden's original criterion when a fractional Brownian motion with Hurst index $H$ adopts a polynomial drift term with exponent $H+\frac{1}{2}$.
\end{abstract}

Keywords: CUSUM; sequential change detection; change-point detection; fractional Brownian motion; fractional Ornstein-Uhlenbeck; diffusion-type process; optimality

2010 Mathematics Subject Classification: Primary 60G35; 60G22

Secondary 60L10; 60G40

\section{Introduction}

The quick detection of an abrupt change in the behavior of a stochastic system is an important problem in many application areas, such as quality control, target tracking, navigation, seismology, biosurveillance, and computer security. Specifically, the problem is to find a detection rule that raises an alarm as soon as possible after the change has occurred based on sequential observations of the system. Thus, a good detection rule should have small detection delay, but also low frequency of false alarms in its repeated applications.

There are three main formulations that balance the trade-off between these two antithetic goals. A Bayesian approach, developed by Shiryaev [29], where the change point is modeled as a random variable, and two minimax approaches, due to Lorden [14] and Pollak [23], where the change point is considered to be unknown, but deterministic. For a comparison of these formulations, we refer the reader to Moustakides [19]. For exhaustive treatments of sequential change detection, we refer the reader to the books of Basseville and Nikiforov [1] and Hadjiliadis and Poor [8].

Lorden's approach has a deep connection with the cumulative sum (CUSUM) test, a detection structure that was proposed by Page [22] and is very popular in applications (see, for example, [9]). In particular, Lorden [14] quantified the performance of a detection rule with its worst (with respect to the time of the change) conditional expected detection delay given the worst possible scenario until the time of the change and suggested the minimization of this criterion subject to an upper bound on the rate of false alarms. In the case of independent and

Received 8 February 2011; revision received 12 August 2012.

* Postal address: Department of Statistics and Applied Probability, University of California, Santa Barbara, CA 93106, USA. Email address: chronopoulou@pstat.ucsb.edu

** Postal address: Department of Mathematics, 3620 South Vermont Avenue, Los Angeles, CA 90089, USA.

Email address: fellouri@usc.edu 
identically distributed (i.i.d.) observations before and after the change, Lorden [14] showed that the CUSUM test is an asymptotic solution to this problem as the rate of false alarms goes to 0. It was later shown by Moustakides [17] (see also Ritov [27]) that the CUSUM test is an exact solution to Lorden's optimization problem in the case of i.i.d. observations. This exact optimality property was extended in continuous time by Shiryaev [30] and Beibel [2] in the case that a standard Brownian motion adopts a linear drift. Moustakides [18] generalized this result by showing that the CUSUM procedure optimizes a modified version of Lorden's criterion for the problem of detecting a change in the (random) drift of a diffusion-type process.

In the present work we show that the CUSUM test is optimal with respect to the criterion considered in [18] for detecting a change in the distribution of an arbitrary continuous-path process. Moreover, we apply this general result to the case that a (random) drift emerges in a fractional process of diffusion type. We show in particular that the CUSUM test is optimal for detecting a change from a fractional Brownian motion (FBM) to a fractional OrnsteinUhlenbeck (FOU) process, as well as for detecting the emergence of linear drift in an FBM. The latter optimality properties are valid for any value of the Hurst index, $H$, which characterizes the pathwise and distributional properties of FBM. As a by-product, we establish the optimality of the CUSUM test with respect to Lorden's original criterion when an FBM with Hurst index $H$ adopts a polynomial drift term with exponent $H+\frac{1}{2}$. In this way, we generalize the optimality of the CUSUM for the detection of linear drift in standard Brownian motion, which corresponds to the special case $H=\frac{1}{2}$.

The previous optimality properties are not in the scope of existing results, since FBM is not a semimartingale unless $H=\frac{1}{2}$, in which case it reduces to standard Brownian motion. However, FBM is well suited for the description of phenomena that are characterized by self-similarity and/or long memory, which explains the fact that it has been used as the basic building block for models in a variety of fields, such as hydrology, traffic networks, finance, and economics (see, for example, [3], [5], [6], and [11]).

These diverse applications have also triggered a great interest in the statistical inference for processes related to FBM. In particular, Kleptsyna and Le Breton [12] studied the properties of the maximum likelihood estimator (MLE) for the parameter in the drift of an FOU process with Hurst index $H>\frac{1}{2}$. Tudor and Viens [31] used Malliavin calculus techniques to study the properties of the MLE for any $H \in(0,1)$ and a more general class of fractional diffusions, in which the drift coefficient is linear with respect to the unknown parameter. For the same class of processes, Rao [24] considered a sequential version of the MLE. The problem of sequential testing for fractional diffusion-type processes was considered by Rao [25].

The rest of this paper is organized as follows. In Section 2 we formulate the problem and establish the optimality of the CUSUM test for arbitrary continuous-path stochastic processes. In Section 3 we apply this general result to the case of fractional diffusion-type processes. We conclude in Section 4.

\section{CUSUM optimality for continuous-path processes}

\subsection{Problem formulation}

Let $(\Omega, \mathcal{F})$ be the canonical space of continuous functions on $[0, \infty)$ that vanish at 0 . We denote by $\left\{\xi_{t}\right\}$ the coordinate process on this space and by $\left\{\mathcal{F}_{t}\right\}$ the right-continuous version of its natural filtration. Thus, $\xi_{t}(\omega):=\omega(t)$ for every $\omega \in \Omega$ and $\mathcal{F}_{t}:=g_{t+}$, where $g_{t}:=$ $\sigma\left(\xi_{s}: 0 \leq s \leq t\right)$ for every $t>0$. 
Let $\mathbb{P}_{0}$ and $\mathbb{P}_{\infty}$ be two completely specified probability measures on $(\Omega, \mathcal{F})$. We assume that $\mathbb{P}_{0}$ and $\mathbb{P}_{\infty}$ are locally equivalent, i.e. they are mutually absolutely continuous on $\mathcal{F}_{t}$ for any $0<t<\infty$, and we denote by $\left\{u_{t}\right\}$ the log-likelihood ratio process

$$
u_{t}:=\left.\log \frac{\mathrm{d} \mathbb{P}_{0}}{\mathrm{~d} \mathbb{P}_{\infty}}\right|_{\mathcal{F}_{t}}, \quad t>0 ; \quad u_{0}:=1 .
$$

We assume that the distribution of $\left\{\xi_{t}\right\}$, which we denote by $\mathbb{P}_{\tau}$, changes at some unknown, deterministic time $\tau \in[0, \infty]$ from $\mathbb{P}_{\infty}$ to $\mathbb{P}_{0}$. This means that $\mathbb{P}_{\tau}$ coincides with $\mathbb{P}_{\infty}$ on $\mathcal{F}_{t}$ for $t \in[0, \tau]$, whereas it is locally equivalent to $\mathbb{P}_{\infty}$ on $\mathcal{F}_{t}$ for $t>\tau$. In particular,

$$
u_{t}-u_{\tau}=\left.\log \frac{\mathrm{d} \mathbb{P}_{\tau}}{\mathrm{d} \mathbb{P}_{\infty}}\right|_{\mathscr{F}_{t}}, \quad t>\tau .
$$

A sequential detection rule $T$ is an $\left\{\mathscr{F}_{t}\right\}$-stopping time, at which we stop and declare that the change has occurred. An ideal detection rule should take large values under $\mathbb{P}_{\infty}$, while minimizing detection delay in some meaningful sense for every possible change point $\tau \in$ $[0, \infty)$. Since this is not possible for every $\tau$, we take a minimax approach and consider the constrained optimization problem

$$
\begin{gathered}
\inf _{T} \mathscr{g}_{M}[T] \text { when } \frac{1}{2} \mathbb{E}_{\infty}\left[\langle u\rangle_{T}\right] \geq \gamma, \\
\text { where } \mathscr{g}_{M}[T]:=\sup _{\tau \geq 0} \operatorname{esssup} \frac{1}{2} \mathbb{E}_{\tau}\left[\left(\langle u\rangle_{T}-\langle u\rangle_{\tau}\right)^{+} \mid \mathcal{F}_{\tau}\right],
\end{gathered}
$$

where $\langle u\rangle_{t}$ is the quadratic variation of $u_{t}$ and $x^{+}:=\max \{x, 0\}$. When the observed process is of diffusion type, (1) reduces to the optimization problem considered in [18]. When $\langle u\rangle_{t}$ is proportional to $t,(1)$ is equivalent to Lorden's optimization problem:

$$
\begin{gathered}
\inf _{T} \mathscr{g}_{L}[T] \text { when } \mathbb{E}_{\infty}[T] \geq \gamma, \\
\text { where } \quad \mathscr{g}_{L}[T]:=\sup _{\tau \geq 0} \operatorname{esssup} \mathbb{E}_{\tau}\left[(T-\tau)^{+} \mid \mathcal{F}_{\tau}\right] .
\end{gathered}
$$

In both problems, $\gamma$ is a fixed, positive number that is chosen by the designer of the scheme depending on her tolerance to false alarms for the particular application of interest. We will say that a detection rule is $g_{M}$-optimal or $\mathscr{g}_{L}$-optimal if it solves the problem defined in (1) or, respectively, (2) for an arbitrary value of $\gamma$.

In both formulations, the goal is to find a detection rule that minimizes the worst (with respect to $\tau$ ) conditional expected detection delay given the worst possible history of observations up to the time of the change subject to a lower bound, $\gamma$, on the period of false alarms. However, detection delay and period of false alarms are measured in terms of the actual time in (2) and in terms of the accumulated quadratic variation of the log-likelihood ratio process in (1). The main advantage of the latter formulation is its tractability, which leads to a simple optimizer for a large class of processes, as we will see in the next subsection where we present our main result.

\subsection{Main result}

Let us first define the CUSUM stopping time

$$
S_{c}:=\inf \left\{t \geq 0: y_{t} \geq c\right\}, \quad \text { where } \quad y_{t}:=u_{t}-\inf _{0 \leq s \leq t} u_{s}, t \geq 0,
$$

and $c$ is a positive constant that is chosen to satisfy the relevant false alarm constraint with equality. Specifically, $c$ is chosen so that $\mathbb{E}_{\infty}\left[\langle u\rangle_{S_{c}}\right]=\gamma$ for the problem defined in (1) and 
$\mathbb{E}_{\infty}\left[S_{c}\right]=\gamma$ for the problem defined in (2). Then, it is known from [18] that the CUSUM test is $g_{M}$-optimal for detecting a change in the random drift of a diffusion-type process that satisfies a 'full-energy' condition. If, additionally, the observed diffusion-type process has constant 'signal-to-noise ratio' before and after the change, as is the case when a linear drift emerges in a standard Brownian motion, then the CUSUM test is also $g_{L}$-optimal. With the following theorem we extend these optimality properties to a larger class of dynamics.

Theorem 1. The CUSUM test is $\mathrm{g}_{M}$-optimal if

$$
\lim _{t \rightarrow \infty}\langle u\rangle_{t}=\infty \quad \mathbb{P}_{0}, \mathbb{P}_{\infty} \text {-almost surely. }
$$

When, in particular, $\langle u\rangle_{t}$ is proportional to $t$, the CUSUM test is also $g_{L}$-optimal.

The proof of this theorem relies on the technique introduced in [18] and the following lemma, which reveals the structure of the log-likelihood ratio process $\left\{u_{t}\right\}$.

Lemma 1. There exist continuous processes $\left\{\tilde{X}_{t}\right\}$ and $\left\{X_{t}\right\}$, which are local martingales (vanishing at 0 ) with respect to $\mathbb{P}_{\infty}$ and $\mathbb{P}_{0}$, respectively, so that

$$
u_{t}=\tilde{X}_{t}-\frac{1}{2}\langle u\rangle_{t}=-X_{t}+\frac{1}{2}\langle u\rangle_{t}, \quad t \geq 0 .
$$

Proof. Since the likelihood ratios $\left\{\mathrm{e}^{u_{t}}\right\}$ and $\left\{\mathrm{e}^{-u_{t}}\right\}$ are continuous martingales with respect to $\mathbb{P}_{\infty}$ and $\mathbb{P}_{0}$, respectively, it is well known (see, for example, Proposition 1.6 of [26, p. 328]) that there exist unique, continuous, local martingales $\left\{\tilde{X}_{t}\right\}$ and $\left\{X_{t}\right\}$ with respect to $\mathbb{P}_{\infty}$ and $\mathbb{P}_{0}$, respectively, so that

$$
\mathrm{e}^{u_{t}}=\exp \left\{\tilde{X}_{t}-\frac{1}{2}\langle\tilde{X}\rangle_{t}\right\}, \quad \mathrm{e}^{-u_{t}}=\exp \left\{X_{t}-\frac{1}{2}\langle X\rangle_{t}\right\},
$$

where $X_{0}=\tilde{X}_{0}=0$. Taking logarithms we obtain

$$
u_{t}=\tilde{X}_{t}-\frac{1}{2}\langle\tilde{X}\rangle_{t}=-X_{t}+\frac{1}{2}\langle X\rangle_{t},
$$

and, consequently, $\langle u\rangle_{t}=\langle\tilde{X}\rangle_{t}=\langle X\rangle_{t}$, which completes the proof.

This lemma has some important ramifications. First, from an application of Wald's identity, it follows that, for any stopping time $T$ with $\mathbb{E}_{i}\left[\langle u\rangle_{T}\right]<\infty$ for $i=0, \infty$,

$$
\frac{1}{2} \mathbb{E}_{\infty}\left[\langle u\rangle_{T}\right]=\mathbb{E}_{\infty}\left[-u_{T}\right]=\mathbb{E}_{\infty}\left[\left.\log \frac{\mathrm{d} \mathbb{P}_{\infty}}{\mathrm{d} \mathbb{P}_{0}}\right|_{\mathcal{F}_{T}}\right]
$$

and that, on the event $\{T>\tau\}$,

$$
\frac{1}{2} \mathbb{E}_{\tau}\left[\langle u\rangle_{T}-\langle u\rangle_{\tau} \mid \mathcal{F}_{\tau}\right]=\mathbb{E}_{\tau}\left[u_{T}-u_{\tau} \mid \mathcal{F}_{\tau}\right]=\mathbb{E}_{\tau}\left[\left.\log \frac{\mathrm{d} \mathbb{P}_{\tau}}{\mathrm{dP} \mathbb{P}_{\infty}}\right|_{\mathcal{F}_{T}} \mid \mathscr{F}_{\tau}\right],
$$

which highlights the connection of (1) with the notion of Kullback-Leibler information and justifies calling (as in [18]) $\mathscr{g}_{M}[T]$ the $K-L$ detection divergence and $\frac{1}{2} \mathbb{E}_{\infty}\left[\langle u\rangle_{T}\right]$ the $K-L$ false alarm divergence of $T$.

Second, and most importantly, Lemma 1 allows us to obtain closed-form expressions for the performance characteristics of the CUSUM rule and establish its optimality along the lines of [18]. 
Lemma 2. Suppose that condition (3) holds. For any $c>0, \tau \in[0, \infty)$, and stopping time $T$,

$$
\mathbb{P}_{\tau}\left(T_{c}<\infty\right)=1, \quad \mathbb{P}_{\infty}\left(T_{c}<\infty\right)=1,
$$

where $T_{c}:=T \wedge S_{c}=\min \left(T, S_{c}\right)$. Moreover, on the event $\left\{T_{c}>\tau\right\}$, we have

$$
\begin{aligned}
\frac{1}{2} \mathbb{E}_{\tau}\left[\langle u\rangle_{T_{c}}-\langle u\rangle_{\tau} \mid \mathcal{F}_{\tau}\right] & =\mathbb{E}_{\tau}\left[g\left(y_{T_{c}}\right)-g\left(y_{\tau}\right) \mid \mathcal{F}_{\tau}\right], \\
\frac{1}{2} \mathbb{E}_{\infty}\left[\langle u\rangle_{T_{c}}-\langle u\rangle_{\tau} \mid \mathcal{F}_{\tau}\right] & =\mathbb{E}_{\infty}\left[h\left(y_{T_{c}}\right)-h\left(y_{\tau}\right) \mid \mathcal{F}_{\tau}\right],
\end{aligned}
$$

where the functions $g$ and $h$ are defined as

$$
g(x):=\mathrm{e}^{-x}+x-1, \quad h(x):=\mathrm{e}^{x}-x-1, \quad x \geq 0 .
$$

Proof. Let us introduce the following notation:

$$
T_{c}^{n}:=T_{c} \wedge \inf \left\{t \geq 0:\langle u\rangle_{t} \geq n\right\}, \quad n \in \mathbb{N} .
$$

From an application of Itô's rule we have

$$
g\left(y_{T_{c}^{n}}\right)-g\left(y_{\tau}\right)=\int_{\tau}^{T_{c}^{n}} g^{\prime}\left(y_{s}\right) \mathrm{d} y_{s}+\frac{1}{2} \int_{\tau}^{T_{c}^{n}} g^{\prime \prime}\left(y_{s}\right) \mathrm{d}\langle y\rangle_{s} .
$$

From Lemma 1 and the definition of the CUSUM statistic $\left\{y_{t}\right\}$, we have

$$
y_{t}=u_{t}-m_{t}=-X_{t}+\frac{1}{2}\langle u\rangle_{t}-m_{t}, \quad \text { where } \quad m_{t}:=\inf _{0 \leq s \leq t} u_{s},
$$

and, consequently, $\langle y\rangle_{t}=\langle u\rangle_{t}$; therefore, we can write

$$
g\left(y_{T_{c}^{n}}\right)-g\left(y_{\tau}\right)=\int_{\tau}^{T_{c}^{n}} g^{\prime}\left(y_{s}\right)\left[-\mathrm{d} X_{s}+\frac{\mathrm{d}\langle u\rangle_{s}}{2}-\mathrm{d} m_{s}\right]+\frac{1}{2} \int_{\tau}^{T_{c}^{n}} g^{\prime \prime}\left(y_{s}\right) \mathrm{d}\langle u\rangle_{s} .
$$

Now, with a rearrangement of the right-hand side and using the fact that the measure $\mathrm{d} m_{s}$ is carried by the set $\left\{y_{s}=0\right\}$, the previous relationship takes the following form:

$$
g\left(y_{T_{c}^{n}}\right)-g\left(y_{\tau}\right)=\frac{1}{2} \int_{\tau}^{T_{c}^{n}}\left(g^{\prime}+g^{\prime \prime}\right)\left(y_{s}\right) \mathrm{d}\langle u\rangle_{s}-\int_{\tau}^{T_{c}^{n}} g^{\prime}\left(y_{s}\right) \mathrm{d} X_{s}-\int_{\tau}^{T_{c}^{n}} g^{\prime}(0) \mathrm{d} m_{s} .
$$

From (6), it is clear that $g^{\prime}(x)+g^{\prime \prime}(x)=1$ and $g^{\prime}(0)=0$; thus,

$$
g\left(y_{T_{c}^{n}}\right)-g\left(y_{\tau}\right)=\frac{1}{2} \int_{\tau}^{T_{c}^{n}} \mathrm{~d}\langle u\rangle_{s}-\int_{\tau}^{T_{c}^{n}} g^{\prime}\left(y_{S}\right) \mathrm{d} X_{s} .
$$

Taking the conditional expectation under $\mathbb{P}_{\tau}$ given $\mathcal{F}_{\tau}$ on the event $\left\{T_{c}>\tau\right\}$ we have

$$
\mathbb{E}_{\tau}\left[g\left(y_{T_{c}^{n}}\right)-g\left(y_{\tau}\right) \mid \mathcal{F}_{\tau}\right]=\mathbb{E}_{\tau}\left[\frac{1}{2} \int_{\tau}^{T_{c}^{n}} \mathrm{~d}\langle u\rangle_{s} \mid \mathcal{F}_{\tau}\right]-\mathbb{E}_{\tau}\left[\int_{\tau}^{T_{c}^{n}} g^{\prime}\left(y_{s}\right) \mathrm{d} X_{S} \mid \mathcal{F}_{\tau}\right] .
$$

Since $0 \leq y_{s} \leq c$ on $\left\{\tau<s<T_{c}^{n} \leq T_{c}\right\}$ and $g^{\prime}$ is an increasing function on [0, $\infty$ ), we have $g^{\prime}\left(y_{s}\right) \leq g^{\prime}(c)$. Moreover, by Lemma 1 and the definition of the stopping time $T_{c}^{n}$, it is clear that $\langle X\rangle_{T_{c}^{n}}=\langle u\rangle_{T_{c}^{n}} \leq n$. Therefore, on $\left\{T_{c}>\tau\right\}$, we have

$$
\int_{\tau}^{T_{c}^{n}}\left(g^{\prime}\left(y_{s}\right)\right)^{2} \mathrm{~d}\langle X\rangle_{s} \leq\left(g^{\prime}(c)\right)^{2}\langle X\rangle_{T_{c}^{n}} \leq\left(g^{\prime}(c)\right)^{2} n<\infty,
$$


which implies that the second term on the right-hand side of (7) vanishes. Consequently, on the event $\left\{T_{c}>\tau\right\}$, we can write

$$
\mathbb{E}_{\tau}\left[g\left(y_{T_{c}^{n}}\right)-g\left(y_{\tau}\right) \mid \mathcal{F}_{\tau}\right]=\mathbb{E}_{\tau}\left[\frac{1}{2} \int_{\tau}^{T_{c}^{n}} \mathrm{~d}\langle u\rangle_{s} \mid \mathcal{F}_{\tau}\right]
$$

Since $g$ is an increasing function on $[0, \infty)$ and $y_{T_{c}^{n}} \in[0, c]$, it is clear that the left-hand side of this equality is bounded by $g(c)-g(0)=g(c)$. Moreover, due to condition (3), $T_{c}^{n}$ converges to $T_{c}, \mathbb{P}_{0}$-almost surely as $n \rightarrow \infty$. Therefore, letting $n \rightarrow \infty$ on the right-hand side of (8), from the monotone convergence theorem we obtain

$$
g(c) \geq \mathbb{E}_{\tau}\left[\frac{1}{2} \int_{\tau}^{T_{c}} \mathrm{~d}\langle u\rangle_{s} \mid \mathcal{F}_{\tau}\right] \geq \mathbb{E}_{\tau}\left[\frac{1}{2} \mathbf{1}_{\left\{T_{c}=\infty\right\}} \int_{\tau}^{\infty} \mathrm{d}\langle u\rangle_{s} \mid \mathcal{F}_{\tau}\right] .
$$

From (3) we have $\mathbb{P}_{0}\left(\int_{\tau}^{\infty} \mathrm{d}\langle u\rangle_{s}=\infty\right)=1$ and, consequently, $\mathbb{P}_{\tau}\left(\int_{\tau}^{\infty} \mathrm{d}\langle u\rangle_{s}=\infty\right)=1$. Then, from the law of the iterated expectation we obtain

$$
\mathbb{E}_{\tau}\left[\mathbb{P}_{\tau}\left(\int_{\tau}^{\infty} \mathrm{d}\langle u\rangle_{s}=\infty \mid \mathcal{F}_{\tau}\right)\right]=1,
$$

which implies that $\mathbb{P}_{\tau}\left(\int_{\tau}^{\infty} \mathrm{d}\langle u\rangle_{s}=\infty \mid \mathcal{F}_{\tau}\right)=1$. Therefore, $\mathbb{P}_{\tau}\left(T_{c}=\infty\right)=0$; otherwise, the right-hand side of (9) becomes infinite due to condition (3) and this leads to a contradiction. Finally, letting $n \rightarrow \infty$ on both sides of (8) simultaneously, application of the bounded convergence and monotone convergence theorems to the left- and right-hand sides, respectively, yield the first relationship in (6). The second relationship in (6) and the fact that $\mathbb{P}_{\infty}\left(T_{c}=\infty\right)=0$ can be shown in a similar way, completing the proof.

If we set $T=S_{c}$ in Lemma 2, we obtain interesting information regarding the behavior of the CUSUM test. First, from (4) it follows that $S_{c}$ terminates almost surely for any change point $\tau \in[0, \infty)$, as well as when the change never occurs $(\tau=\infty)$, i.e. it is almost certain that the CUSUM test will raise a false alarm.

Furthermore, the first relationship in (5) implies that, on the event $\left\{S_{c}>\tau\right\}$, we have

$$
\frac{1}{2} \mathbb{E}_{\tau}\left[\langle u\rangle_{S_{c}}-\langle u\rangle_{\tau} \mid \mathcal{F}_{\tau}\right]=\mathbb{E}_{\tau}\left[g\left(y_{S_{c}}\right)-g\left(y_{\tau}\right) \mid \mathcal{F}_{\tau}\right]=g(c)-g\left(y_{\tau}\right),
$$

where the last equation is due to the fact that $y_{S_{c}}=c$, which follows from the continuity of the paths of the CUSUM process, $\left\{y_{t}\right\}$. Therefore, since $y_{\tau} \in[0, c)$ on $\left\{S_{c}>\tau\right\}$ and $g$ is an increasing function on $[0, \infty)$, it follows that

$$
\begin{aligned}
\mathscr{g}_{M}\left[S_{c}\right] & =\sup _{\tau \geq 0} \operatorname{esssup} \frac{1}{2} \mathbb{E}_{\tau}\left[\left(\langle u\rangle_{S_{c}}-\langle u\rangle_{\tau}\right) \mathbf{1}_{\left\{S_{c}>\tau\right\}} \mid \mathcal{F}_{\tau}\right] \\
& =\sup _{\tau \geq 0} \operatorname{esssup} \mathbb{E}_{\tau}\left[\left(g(c)-g\left(y_{\tau}\right)\right) \mathbf{1}_{\left\{S_{c}>\tau\right\}} \mid \mathcal{F}_{\tau}\right] \\
& =g(c) \\
& =\frac{1}{2} \mathbb{E}_{0}\left[\langle u\rangle_{S_{c}}\right] .
\end{aligned}
$$

The last equality follows by setting $\tau=0$ in (10) and implies that the worst-case scenario for the CUSUM test is when the change occurs from the beginning, i.e. at $\tau=0$. Finally, setting $\tau=0$ in the second relationship in (5) yields

$$
\frac{1}{2} \mathbb{E}_{\infty}\left[\langle u\rangle_{S_{c}}\right]=\mathbb{E}_{\infty}\left[h\left(y_{S_{c}}\right)\right]=h(c) .
$$


Therefore, for the CUSUM test to satisfy the false alarm constraint in (1) with equality, its threshold $c$ must be selected as the (unique) solution to the nonlinear equation $h(c)=\gamma$. The following lemma states that we can actually restrict ourselves to stopping times that satisfy the false alarm constraint with equality.

Lemma 3. Suppose that condition (3) holds. For any stopping time $T$ such that $\frac{1}{2} \mathbb{E}_{\infty}\left[\langle u\rangle_{T}\right]>$ $\gamma$, there exists a $c^{\prime}>0$ such that $\frac{1}{2} \mathbb{E}_{\infty}\left[\langle u\rangle_{T_{c^{\prime}}}\right]=\gamma$ and $\mathscr{g}_{M}\left[T_{c^{\prime}}\right] \leq \mathscr{g}_{M}[T]$.

Proof. Consider an arbitrary stopping time $T$ with $\frac{1}{2} \mathbb{E}_{\infty}\left[\langle u\rangle_{T}\right]>\gamma$, and define the function $\psi(c):=\frac{1}{2} \mathbb{E}_{\infty}\left[\langle u\rangle_{T_{c}}\right], c>0$. From the second relationship in (5), it follows that $\psi(c)=$ $\mathbb{E}_{\infty}\left[h\left(y_{T_{c}}\right)\right]$, which implies that $\psi(c)$ is a continuous function. Then, since $\psi(0)=0$ and $\psi(\infty)=\frac{1}{2} \mathbb{E}_{\infty}\left[\langle u\rangle_{T}\right]>\gamma$, there exists some $c^{\prime}>0$ such that $\psi\left(c^{\prime}\right)=\gamma$. Since $T_{c^{\prime}}=$ $T \wedge S_{\mathcal{C}^{\prime}} \leq T$, this implies that $\mathscr{g}_{M}\left[T_{\mathcal{C}^{\prime}}\right] \leq \mathscr{g}_{M}[T]$, completing the proof.

Proof of Theorem 1. The proof is based on the fact that, for any stopping time $T$ and $c>0$, we have the lower bound

$$
g_{M}[T] \geq \frac{\mathbb{E}_{\infty}\left[\mathrm{e}^{y_{T_{c}}} g\left(y_{T_{c}}\right)\right]}{\mathbb{E}_{\infty}\left[\mathrm{e}^{y_{T_{C}}}\right]},
$$

which can be shown in exactly the same way as in Theorem 2 of [18]. From (11) and the fact that $y_{S_{c}}=c$, it follows that both sides of the above inequality equal $g(c)$ when $T=S_{c}$. Due to this observation and Lemma 3, it suffices to show that

$$
g(c)=\inf _{T} \frac{\mathbb{E}_{\infty}\left[\mathrm{e}^{y_{T_{c}}} g\left(y_{T_{c}}\right)\right]}{\mathbb{E}_{\infty}\left[\mathrm{e}^{y_{T_{c}}}\right]} \text { when } \frac{1}{2} \mathbb{E}_{\infty}\left[\langle u\rangle_{T}\right]=\gamma .
$$

This can be shown in exactly the same way as in Theorem 3 of [18].

\subsection{The case of diffusion-type processes}

Let us now illustrate Theorem 1 in the case that $\left\{\xi_{t}\right\}$ is a standard Brownian motion that adopts a (random) drift. Specifically, let $\mathbb{P}_{\infty}$ be the Wiener measure and suppose that the post-change measure $\mathbb{P}_{0}$ is induced by the dynamics

$$
\xi_{t}=W_{t}+\int_{0}^{t} \mu_{s} \mathrm{~d} s, \quad t \geq 0,
$$

where $\left\{W_{t}\right\}$ is a standard Brownian motion under $\mathbb{P}_{0}$ and $\left\{\mu_{t}\right\}$ is an $\left\{\mathcal{F}_{t}\right\}$-adapted process that satisfies

$$
\begin{gathered}
\mathbb{P}_{\infty}\left(\int_{0}^{t} \mu_{s}^{2} \mathrm{~d} s<\infty\right)=1, \quad t \geq 0, \\
\mathbb{E}_{\infty}\left[\exp \left\{\int_{0}^{t} \mu_{s} \mathrm{~d} \xi_{s}-\frac{1}{2} \int_{0}^{t} \mu_{s}^{2} \mathrm{~d} s\right\}\right]=1, \quad t \geq 0 .
\end{gathered}
$$

These two conditions guarantee that $\mathbb{P}_{0}$ is indeed well defined and locally equivalent to the Wiener measure, $\mathbb{P}_{\infty}$. Moreover, from Girsanov's theorem we obtain the following representation for their log-likelihood ratio:

$$
u_{t}=\int_{0}^{t} \mu_{s} \mathrm{~d} \xi_{s}-\frac{1}{2} \int_{0}^{t} \mu_{s}^{2} \mathrm{~d} s, \quad t \geq 0 .
$$

It is then clear that $\langle u\rangle_{t}=\int_{0}^{t} \mu_{s}^{2} \mathrm{~d} s$ and Theorem 1 implies that the CUSUM test is $g_{M}$-optimal if, additionally, the following condition is satisfied:

$$
\int_{0}^{\infty} \mu_{s}^{2} \mathrm{~d} s=\infty \quad \mathbb{P}_{0}, \mathbb{P}_{\infty} \text {-almost surely. }
$$


This is exactly the result obtained in [18]. When the process $\left\{\mu_{t}\right\}$ reduces to a constant, $\langle u\rangle_{t}$ is proportional to $t$ and Theorem 1 implies that the CUSUM test is additionally $\mathscr{g}_{L}$-optimal, which is also a known result. However, Theorem 1 is a nontrivial generalization of existing optimality results, as there are many continuous-path processes that are not of diffusion type, not even semimartingales. We illustrate this point in the next section, where we apply Theorem 1 in the context of fractional diffusion-type processes.

\section{The case of fractional diffusion-type processes}

Let us first define FBM, the basic building block of fractional diffusion-type processes, and present its main properties.

\subsection{FBM: a quick review}

A stochastic process $\left\{B_{t}^{H}\right\}$ is an FBM with Hurst index $H \in(0,1)$ if it is a centered, continuous, Gaussian process with covariance structure

$$
\mathbb{E}\left[B_{t}^{H} B_{s}^{H}\right]=\frac{1}{2}\left(t^{2 H}+s^{2 H}-|t-s|^{2 H}\right), \quad t, s \geq 0,
$$

where $\mathbb{E}[\cdot]$ refers to the expectation with respect to the underlying probability measure. As a consequence of its definition, the following properties of FBM hold.

(i) FBM is $H$-self-similar, in the sense that $\left\{B_{t}^{H}\right\}_{t \geq 0}$ has the same finite-dimensional distributions as $\left\{c^{-H} B_{c t}^{H}\right\}_{t \geq 0}$ for every $c>0$.

(ii) FBM has stationary increments, which are independent only when $H=\frac{1}{2}$. When $H<\frac{1}{2}$, they are negatively correlated and the process exhibits short-range dependence, in the sense that

$$
\sum_{n=1}^{\infty} \mathbb{E}\left[\left(B_{n}^{H}-B_{n-1}^{H}\right) B_{1}^{H}\right]<\infty ;
$$

when $H>\frac{1}{2}$, they are positively correlated and the process exhibits long-range dependence, in the sense that

$$
\sum_{n=1}^{\infty} \mathbb{E}\left[\left(B_{n}^{H}-B_{n-1}^{H}\right) B_{1}^{H}\right]=\infty .
$$

(iii) FBM has Hölder continuous paths of order $H-\varepsilon$ for every $0<\varepsilon<H$.

(iv) $\mathrm{FBM}$ has finite $1 / H$-variation (in an $L^{1}$ sense) that is equal to $c_{H}^{\prime} t$ on any finite interval $[0, t]$, where

$$
c_{H}^{\prime}:=\int_{\mathbb{R}}|x|^{1 / H} \phi(x) \mathrm{d} x, \quad \phi(x):=\frac{1}{\sqrt{2 \pi}} \mathrm{e}^{-x^{2} / 2} .
$$

(v) FBM is not a semimartingale, i.e. it does not admit a decomposition as the sum of a local martingale and a term of finite variation (see, for example, [28]). However, the transformed process

$$
M_{t}^{H}:=\int_{0}^{t} k_{H}(t, s) \mathrm{d} B_{s}^{H}, \quad t \geq 0,
$$


is a square-integrable martingale with quadratic variation $\left\langle M^{H}\right\rangle_{t}=\lambda_{H}^{-1} t^{2-2 H}$, where

$$
k_{H}(t, s):=c_{H}^{-1} s^{1 / 2-H}(t-s)^{1 / 2-H}, \quad 0 \leq s \leq t,
$$

$c_{H}$ and $\lambda_{H}$ are positive constants defined as

$$
c_{H}:=2 H \Gamma\left(\frac{3}{2}-H\right) \Gamma\left(H+\frac{1}{2}\right), \quad \lambda_{H}:=\frac{2 H \Gamma(3-2 H) \Gamma(H+1 / 2)}{\Gamma(3 / 2-H)},
$$

and $\Gamma(x):=\int_{0}^{\infty} s^{x-1} \mathrm{e}^{-s} \mathrm{~d} s$ is the gamma function. This result was shown by Molchan [16] and, more recently, by Norros et al. [20], where the process $M^{H}$ was called the fundamental martingale associated with FBM.

For an exhaustive treatment on the properties of FBM, we refer the reader to Chapter 5 of Nualart [21]. Here, we will only add an extension of Lévy's characterization theorem that was recently established by $\mathrm{Hu}$ et al. [10] (see also Mishura and Valkeila [15]), according to which properties (iii), (iv), and (v) characterize FBM. In particular, if $Y$ is a continuous, centered, square-integrable stochastic process with

(a) Hölder continuous paths of order $H-\varepsilon$ for any $\varepsilon>0$,

(b) finite $1 / H$-quadratic variation (in an $L^{1}$ sense) that is equal to $c_{H}^{\prime} t$ on any interval $[0, t]$, and

(c) the process that is defined as $M^{H}$ in (15) with $B^{H}$ replaced by $Y$ is a martingale, whose quadratic variation when $H>\frac{1}{2}$ is almost surely absolutely continuous with respect to the Lebesgue measure,

then $Y$ is an FBM with Hurst index $H$.

\subsection{Optimality of the CUSUM for fractional diffusion-type processes}

We now return to the change detection problem and focus on the special case that the observed process $\left\{\xi_{t}\right\}$ is an FBM that adopts a random drift. Thus, in what follows we assume that $\left\{\xi_{t}\right\}$ is an FBM with Hurst index $H$ under $\mathbb{P}_{\infty}$, whereas $\mathbb{P}_{0}$ is induced by the dynamics

$$
\xi_{t}=B_{t}^{H}+\int_{0}^{t} \mu_{s} \mathrm{~d} s, \quad t \geq 0,
$$

where $\left\{\mu_{t}\right\}$ is an $\left\{\mathcal{F}_{t}\right\}$-adapted process and $\left\{B_{t}^{H}\right\}$ an FBM with Hurst index $H$ under $\mathbb{P}_{0}$. When $H=\frac{1}{2}$, we recover the context of Subsection 2.3, where we saw that $\mathbb{P}_{0}$ is indeed well defined and locally equivalent to $\mathbb{P}_{\infty}$ when conditions (12) and (13) are satisfied and that the CUSUM test is $g_{M}$-optimal if, additionally, condition (14) holds. Our goal in this section is to obtain analogous conditions when $H \neq \frac{1}{2}$, in which case $\left\{\xi_{t}\right\}$ is not a semimartingale and the classical Girsanov theorem does not apply; thus, the previous conditions are no longer appropriate.

To this end, we work with the transformed process

$$
\zeta_{t}:=\int_{0}^{t} k_{H}(t, s) \mathrm{d} \xi_{s}, \quad t \geq 0
$$

and assume that the paths of $\left\{\mu_{t}\right\}$ are sufficiently smooth so that the process

$$
Q_{t}:=\frac{\mathrm{d}}{\mathrm{d}\langle\zeta\rangle_{t}} \int_{0}^{t} k_{H}(t, s) \mu_{s} \mathrm{~d} s, \quad t \geq 0,
$$


is well defined, where the derivative is understood in the sense of absolute continuity. Note that, since $\left\{\xi_{t}\right\}$ is an FBM under $\mathbb{P}_{\infty}$, from property (v) in the previous subsection, it follows that $\langle\zeta\rangle_{t}=\lambda_{H}^{-1} t^{2-2 H}$ and that $\left\{\zeta_{t}\right\}$ is a square-integrable martingale under $\mathbb{P}_{\infty}$. The latter property allows us to obtain an explicit representation for the log-likelihood ratio process $\left\{u_{t}\right\}$ using Girsanov's theorem, although $\left\{\xi_{t}\right\}$ itself is not a semimartingale. This is a well-known result in fractional stochastic calculus (see, for example, [7] or [13]), which we now prove using the characterization of FBM that we discussed at the end of the previous subsection.

\section{Theorem 2. If the conditions}

$$
\begin{gathered}
\mathbb{P}_{\infty}\left(\int_{0}^{t} Q_{s}^{2} \mathrm{~d}\langle\zeta\rangle_{s}<\infty\right)=1, \quad t \geq 0 \\
\mathbb{E}_{\infty}\left[\exp \left\{\int_{0}^{t} Q_{s} \mathrm{~d} \zeta_{s}-\frac{1}{2} \int_{0}^{t} Q_{s}^{2} \mathrm{~d}\langle\zeta\rangle_{s}\right\}\right]=1, \quad t \geq 0
\end{gathered}
$$

hold, then $\mathbb{P}_{0}$ is locally equivalent to $\mathbb{P}_{\infty}$ and

$$
u_{t}=\int_{0}^{t} Q_{s} \mathrm{~d} \zeta_{s}-\frac{1}{2} \int_{0}^{t} Q_{s}^{2} \mathrm{~d}\langle\zeta\rangle_{s}, \quad t \geq 0 .
$$

Moreover, the CUSUM test is $\mathrm{g}_{M}$-optimal in this context if, additionally,

$$
\int_{0}^{\infty} Q_{s}^{2} \mathrm{~d}\langle\zeta\rangle_{s}=\infty \quad \mathbb{P}_{0}, \mathbb{P}_{\infty} \text {-almost surely. }
$$

Proof. Owing to Theorem 1, we only need to show (19). Specifically, we need to show that if the post-change measure $\mathbb{P}_{0}$ is defined by

$$
\left.\frac{\mathrm{d} \mathbb{P}_{0}}{\mathrm{~d} \mathbb{P}_{\infty}}\right|_{\mathcal{F}_{t}}=\exp \left\{\int_{0}^{t} Q_{s} \mathrm{~d} \zeta_{s}-\frac{1}{2} \int_{0}^{t} Q_{s}^{2} \mathrm{~d}\langle\zeta\rangle_{s}\right\}, \quad t \geq 0,
$$

then the process

$$
B_{t}^{H}:=\xi_{t}-\int_{0}^{t} \mu_{s} \mathrm{~d} s, \quad t \geq 0,
$$

is FBM under $\mathbb{P}_{0}$. From Hu et al. [10], it suffices to show that $\left\{B_{t}^{H}\right\}$ satisfies properties (a), (b), and (c). Since $\left\{B_{t}^{H}\right\}$ is a 'shifted' version of $\left\{\xi_{t}\right\}$, which is FBM under $\mathbb{P}_{\infty}$, it clearly satisfies (a) and (b). It remains to show that the process $\left\{\int_{0}^{t} k_{H}(t, s) \mathrm{d} B_{s}^{H}\right\}$ is a $\mathbb{P}_{0}$-martingale (with absolutely continuous quadratic variation when $H>\frac{1}{2}$ ). Indeed,

$$
\int_{0}^{t} k_{H}(t, s) \mathrm{d} B_{s}^{H}=\int_{0}^{t} k_{H}(t, s)\left(\mathrm{d} \xi_{s}-\mu_{s} \mathrm{~d} s\right)=\zeta_{t}-\int_{0}^{t} Q_{s} \mathrm{~d}\langle\zeta\rangle_{s},
$$

where the second equality is due to (17) and (18). But, from (21) and Girsanov's theorem, it follows that $\left\{\zeta_{t}-\int_{0}^{t} Q_{s} \mathrm{~d}\langle\zeta\rangle_{s}\right\}$ is a $\mathbb{P}_{0}$-martingale with quadratic variation equal to $\langle\zeta\rangle_{t}=$ $\lambda_{H}^{-1} t^{2-2 H}$, which completes the proof.

In what follows, we apply this result to some interesting special cases. 


\subsection{The case of fractional diffusions}

The conditions of Theorem 2 are satisfied for a large class of fractional diffusions and any Hurst index $H$. This is the content of the following corollary, which is based on Lemma 3 of [31].

Corollary 1. The CUSUM test is $g_{M}$-optimal when $\mu_{t}=b\left(\xi_{t}\right)$ and $b$ is a real function so that

(i) $x b(x)$ has a constant sign for all $x>0$ and all $x<0$,

(ii) $|b(x) / x|=c+r(x)$ for all $x$, where $r(x) \rightarrow 0$ as $x \rightarrow \infty$.

These two conditions are satisfied when, for example, $b(x)=c_{0}+c_{1} x+(|x| \wedge 1)^{\alpha}$ with $c_{0}, c_{1} \in \mathbb{R}$ and $\alpha \in[0,1)$. When, in particular, $\alpha=0,\left\{\xi_{t}\right\}$ is an FOU process under the post-change measure $\mathbb{P}_{0}$. This is the fractional analogue of the classical Ornstein-Uhlenbeck process and has recently been used in financial modeling (see, for example, [5] and [6]). For a study of its main properties, we refer the reader to [4].

\subsection{The case of polynomial drift}

When $\mu_{t}=t^{\alpha}$, where $\alpha$ is a real constant, the post-change dynamics (16) reduce to

$$
\xi_{t}=B_{t}^{H}+\int_{0}^{t} s^{\alpha} \mathrm{d} s=B_{t}^{H}+\frac{t^{\alpha+1}}{\alpha+1}
$$

and the process $Q_{t}$ takes the form

$$
Q_{t}=\frac{1}{c_{H}} \frac{\mathrm{d}}{\mathrm{d}\langle\zeta\rangle_{t}} \int_{0}^{t} s^{1 / 2-H+\alpha}(t-s)^{1 / 2-H} \mathrm{~d} s .
$$

Recalling that $\langle\zeta\rangle_{t}=\lambda_{H}^{-1} t^{2-2 H}$, after some algebraic manipulations we obtain

$$
Q_{t}=d_{H, \alpha} t^{\alpha}, \quad \text { where } \quad d_{H, \alpha}:=\frac{\Gamma(3-2 H) \Gamma(3 / 2-H+\alpha)}{\Gamma(3-2 H+\alpha) \Gamma(3 / 2-H)} \frac{2-2 H+\alpha}{2-2 H},
$$

and, consequently,

$$
\int_{0}^{t} Q_{s}^{2} \mathrm{~d}\langle\zeta\rangle_{s}=v_{H, \alpha} t^{2-2 H+2 \alpha}, \quad \text { where } \quad v_{H, \alpha}:=\frac{\left(d_{H, \alpha}\right)^{2}}{\lambda_{H}} \frac{1-H}{1-H+\alpha} .
$$

This expression is the basis for the following corollary.

Corollary 2. If $\mu_{t}=t^{\alpha}$ with $\alpha+1>H$ then the CUSUM test is $g_{M}$-optimal. When, in particular, $\alpha+1=H+\frac{1}{2}$, the CUSUM test is also $\mathscr{g}_{L}$-optimal.

Proof. From (22), it follows that condition (20) is satisfied when $2-2 H+2 \alpha>0$ and that $\langle u\rangle_{t}$ is proportional to $t$ when $2-2 H+2 \alpha=1$. It is now clear that the first claim is a consequence of Theorem 2, whereas the second claim is a consequence of Theorem 1.

Corollary 2 implies that the CUSUM test is $g_{M}$-optimal when a linear $\operatorname{drift}(\alpha=0)$ emerges in FBM for any value of the Hurst index, $H \in(0,1)$. Moreover, it provides a class of processes for which the CUSUM test optimizes Lorden's original criterion. To our knowledge, such an optimality property has been established only for diffusion-type processes (see the discussion on page 313 of [18]). Setting $H=\frac{1}{2}$ we recover the well-known $g_{L}$-optimality of the CUSUM for the detection of linear drift in a standard Brownian motion; thus, Corollary 2 is a generalization of this classical result. 


\subsection{Extensions}

It is possible to generalize Theorem 2 so that the prechange measure $\mathbb{P}_{\infty}$ is induced by the dynamics

$$
\xi_{t}=\int_{0}^{t} \sigma(s) \mathrm{d} \tilde{B}_{s}^{H}, \quad t \geq 0
$$

where $\tilde{B}^{H}$ is an FBM and $\sigma: \mathbb{R}_{+} \rightarrow \mathbb{R}_{+}$is a nonvanishing real function with $\delta$-Hölder continuous paths for some $\delta>1-H$. This smoothness condition guarantees that the integral in (23) can be defined in a Young sense (see [32]). Then, the proof of Theorem 2 carries over as long as we modify the definitions of $\zeta$ and $Q$ in (17) and (18), respectively, as

$$
\zeta_{t}=\int_{0}^{t} k_{H}(t, s) \frac{1}{\sigma(s)} \mathrm{d} \xi_{s}, \quad Q_{t}=\int_{0}^{t} k_{H}(t, s) \frac{\mu_{s}}{\sigma(s)} \mathrm{d} s, \quad t \geq 0 .
$$

Note that, when $\sigma$ is a stochastic process, the integral in (23) cannot be defined in an Itô sense, since FBM is not a semimartingale.

\section{Conclusions}

In this work, the optimality of the CUSUM procedure was established with respect to a modified version of Lorden's criterion for the problem of detecting a change in the distribution of an arbitrary continuous-path process, generalizing existing results that refer to processes of diffusion type. As a consequence, it was shown that the CUSUM test is optimal when a fractional Brownian motion turns into a fractional Ornstein-Uhlenbeck process, or it adopts a linear drift, for any value of the Hurst index, $H$. Furthermore, it was shown that the CUSUM test is optimal with respect to Lorden's original criterion when a fractional Brownian motion with Hurst index $H$ adopts a polynomial drift term with exponent $H+\frac{1}{2}$. In this way, we generalized the well-known optimality of the CUSUM procedure in the case that a linear drift emerges in a standard Brownian motion $\left(H=\frac{1}{2}\right)$.

\section{References}

[1] Basseville, M. And Nikiforov, I. V. (1993). Detection of Abrupt Changes: Theory and Application. PrenticeHall, Engelwood Cliffs, NJ.

[2] Beibel, M. (1996). A note on Ritov's Bayes approach to the minimax property of the cusum procedure. Ann Statist. 24, 1804-1812.

[3] Beran, J. (1994). Statistics for Long-Memory Processes. Chapman and Hall, New York.

[4] Cheridito, P., Kawaguchi, H. and Maejima, M. (2003). Fractional Ornstein-Uhlenbeck processes. Electron. J. Prob. 8, 14pp.

[5] Chronopoulou, A. And Viens, F. G. (2012). Estimation and pricing under long-memory stochastic volatility. Ann. Finance 8, 379-403.

[6] Comte, F. and Renault, E. (1998). Long memory in continuous-time stochastic volatility models. Math. Finance 8, 291-323.

[7] Decreusefond, L. And Üstünel, A. S. (1999). Stochastic analysis of the fractional Brownian motion. Potential Anal. 10, 177-214.

[8] Hadjiliadis, O. and Poor, H. V. (2008). Quickest Detection. Cambridge University Press.

[9] Hawkins, D. M. and Olwell, D. H. (1998). Cumulative Sum Charts and Charting for Quality Improvement. Springer, New York.

[10] Hu, Y., Nualart, D. AND Song, J. (2009). Fractional martingales and characterization of the fractional Brownian motion. Ann. Prob. 37, 2404-2430.

[11] Hurst, H. (1951). Long term storage capacity of reservoirs. Trans. Amer. Soc. Civil Eng. 116, 770-799.

[12] Kleptsyna, M. L. And Le Breton, A. (2002). Statistical analysis of the fractional Ornstein-Uhlenbeck type process. Statist. Infer. Stoch. Process. 5, 229-248. 
[13] Kleptsyna, M. L., Le Breton, A. and Roubaud, M.-C. (2000). Parameter estimation and optimal filtering for fractional type stochastic systems. Statist. Infer. Stoch. Process. 3, 173-182.

[14] Lorden, G. (1971). Procedures for reacting to a change in distribution. Ann. Math. Statist. 42, 1897-1908.

[15] Mishura, J. And Valkeila, E. (2011). An extension of the Lévy characterization to fractional Brownian motion. Ann. Prob. 39, 439-470.

[16] Molchan, G. (1969). Gaussian processes which are asymptotically equivalent to a power of $\lambda$. Theory Prob. Appl. 14, 530-532.

[17] Moustakides, G. V. (1986). Optimal stopping times for detecting changes in distributions. Ann. Statist. 14, 1379-1387.

[18] Moustakides, G. V. (2004). Optimality of the CUSUM procedure in continuous time. Ann. Statist. 32, 302-315.

[19] Moustakides, G. V. (2008). Sequential change detection revisited. Ann. Statist. 2, 787-807.

[20] Norros, I., Valkeila, E. And Virtamo, J. (1999). An elementary approach to a Girsanov formula and other analytical results on fractional Brownian motions. Bernoulli 5, 571-587.

[21] Nualart, D. (2006). The Malliavin Calculus and Related Topics, 2nd edn. Springer, Berlin.

[22] Page, E. S. (1954). Continuous inspection schemes. Biometrika 41, 100-115.

[23] PollaK, M. (1985). Optimal detection of a change in distribution. Ann. Statist. 13, 206-227.

[24] Prakasa Rao, B. L. S. (2004). Sequential estimation for fractional Ornstein-Uhlenbeck type process. Sequent. Anal. 23, 33-44.

[25] Prakasa Rao, B. L. S. (2005). Sequential testing for simple hypotheses for processes driven by fractional Brownian motion. Sequent. Anal. 24, 189-203.

[26] Revuz, D. And Yor, M. (1999). Continuous Martingales and Brownian Motion, 3rd edn. Springer, Berlin.

[27] Ritov, Y. (1990). Decision theoretic optimality of the CUSUM procedure. Ann. Statist. 18, 1464-1469.

[28] Rogers, L. C. G. (1997). Arbitrage with fractional Brownian motion. Math. Finance 7, 95-105.

[29] Shiryaev, A. N. (1978). Optimal Stopping Rules. Springer, New York.

[30] Shiryaev, A. N. (1996). Minimax optimality of the method of cumulative sums (CUSUM) in the continuous time case. Russian Math. Surveys 51, 750-751.

[31] Tudor, C. A. And Viens, F. G. (2007). Statistical aspects of the fractional stochastic calculus. Ann. Statist. 35, 1183-1212.

[32] Young, L. C. (1936). An inequality of the Hölder type, connected with Stieltjes integration. Acta Math. 67, 251-282. 